\title{
Habilidades en Internet de mujeres estudiantes y su relación con la inclusión digital: Nuevas brechas digitales
}

\section{Student Women's Internet Skills and its Relation with the Digital Inclusion: New Digital Divides}

Rocío Jiménez Cortés, Luisa Vega Caro, Alba Vico Bosch

Universidad de Sevilla, España. \{rimenez, luiveg, avico\}@us.es

\section{Resumen}

El objetivo de este trabajo es conocer las habilidades en Internet de las mujeres estudiantes, estableciendo grupos de habilidades más relacionadas con la inclusión digital. Participan en el estudio 215 mujeres estudiantes españolas de 20 a 34 años de edad a través de un proceso de encuesta que incluye escalas validadas para medir las habilidades en Internet y la inclusión digital. Los resultados muestran cuatro perfiles de mujeres estudiantes en función de los tipos de habilidades en Internet y su nivel de desarrollo (principiante "beginner", intermedio "average", avanzado "advanced" y especializado en e-administración). Las habilidades para creary compartir contenidos en redes sociales, así como las habilidades para la e-administración, junto con habilidades para garantizar la privacidad y la seguridad en Internet se relacionan con un grado elevado de inclusión digital en las mujeres estudiantes. Los hallazgos muestran también que las habilidades para la e-administración, y las habilidades para garantizar la seguridad y la privacidad son las que más influyen en la confianza digital. Se observa que las habilidades para crear contenidos culturalmente relevantes y compartirlos en las redes sociales marcan la diferencia fundamental entre el grupo "avanzado" y el grupo "especializado en e-administración". Estos resultados permiten plantear propuestas formativas que prioricen tipos de habilidades para mejorar la inclusión digital en otros perfiles de mujeres y superar nuevas brechas digitales.

\section{Palabras Clave}

Brecha Digital; Alfabetización Digital; Estudios de las Mujeres; Inclusión Digital; Competencias Digitales.

\section{Abstract}

The aims of this research are to know the women's internet skills, and form different groups of skills of women related with the digital inclusion. To do this, we surveyed 215 Spanish student women aged 20 to 34 using two scales measuring their internet skills and digital inclusion. The findings show four profiles of student women depending on the types of Internet skills and their developmental level ("beginner", "average", "advanced" and specialized in e-administration). The skill to create and share content on social networks and skills for e-administration, along with skills to ensure privacy and security on the Internet are associated with a high degree of digital inclusion for student women. The findings showed that the skills for e-administration and the skills to ensure the security and privacy are the most influential in the digital trust. These results suggest the skills to create and share content in social networks sites makes the basic difference between the "advanced" group and the "specialized in e-administration" group. These results raise educational proposals that prioritize skill's types to improve digital inclusion in other profiles of women and overcoming new digital divides.

\section{Keywords}

Digital Divide; Digital Literacy; Women's Studies; Digital Inclusion; Digital Skills. 


\section{Introducción}

Las directrices establecidas por la Agenda Digital Europea 2020 plantean como objetivos la capacitación y la inclusión digitales, con la idea de que la ciudadanía pueda acceder en igualdad de condiciones al espacio común digital europeo. Así, la Agenda Digital fomenta cursos de formación y orientación tecnológica y promueve planes de aprendizaje en los distintos países miembros. Concretamente, en España, el Plan de Inclusión Digital incorpora, como uno de sus ejes de acción, la alfabetización digital. Este eje tiene como objetivo dotar a la población de las competencias digitales básicas que influyan en la calidad de vida y en la empleabilidad, especialmente de determinados sectores poblacionales, entre ellos las mujeres.

Si bien es cierto, los principales informes técnicos sobre la sociedad de la información (Gimeno, 2014) y los indicadores del Eurostat apuntan a que la brecha digital de género se acorta en el acceso a Internet, no obstante, persiste en las formas de uso (van Dijk, 2005) y en las formas de uso avanzado (Menéndez, 2012). En este sentido, la investigación se orienta a profundizar en los diferentes aspectos del proceso de inclusión digital, entre ellos, resulta clave el estudio de las habilidades en Internet (Litt, 2013). Concretamente, en relación al género, la investigación (Correa, 2010; Correa y Jeong, 2011; Hargittai, 2010) ha puesto de manifiesto diferencias en las habilidades que muestran las personas jóvenes en Internet. No obstante, también muestran que cuando se controlan las habilidades de unos y otras, los usos se equiparan ayudando a superar la denominada brecha de participación, especialmente relacionada con la creación de contenidos y su distribución en Internet (Hargittai y Walejko, 2008).

La investigación que presentamos aborda específicamente las habilidades en Internet en el sentido planteado por Helsper y Eynon (2013) y van Deursen y Van Dijk (2010, 2016), es decir, considerando que la combinación y el conjunto de habilidades en Internet conforman la competencia digital. Por tanto, la competencia digital se entiende como un conjunto combinado de habilidades, conocimientos y actitudes específicas de desempeño en el mundo digital. Diferentes autores en nuestro contexto nacional y en el ámbito internacional han propuesto clasificaciones que trascienden los fines técnicos e incorporan habilidades para interactuar con las tecnologías digitales con fines sociales (Area y Pessoa, 2012; Helsper y Eynon, 2013; Kimmons, 2014; Kimmons y Veletsianos, 2014; Lankshear y Knobel, 2008; Livingstone, 2008, Mills, 2010). Así, las aportaciones sobre nuevas alfabetizaciones digitales apuntan a que el impacto de la web 2.0 requiere el aprendizaje de una serie de habilidades que exceden del uso instrumental de dispositivos tecnológicos, recursos y lenguajes informáticos. Estos estudios sobre nuevas alfabetizaciones (New Literacies Studies) tratan de establecer revisiones de las taxonomías de habilidades digitales incorporando no solo la formación instrumental para el uso 
de la tecnología, sino también, y desde enfoques socioculturales, el desarrollo de aspectos cognitivos, emocionales, actitudinales y axiológicos vinculados a su uso (Area y Pessoa, 2012; Aviram y EshetAlkalai 2006; Eshet-Alkalai, 2012; Helsper y Eynon, 2013; Martin y Grudziecki, 2006).

Helsper y Eynon (2013) definen cuatro categorías de habilidades generales: técnica, social, crítica y creativa. Van Deursen y Van Dijk (2010) plantean habilidades operacionales (habilidades para operar con los medios de comunicación digital); formales (habilidades para manejar las estructuras especiales de los medios digitales, como menús y enlaces de hipertexto); informacionales (habilidades para buscar, seleccionar y evaluar la información en formato digital) y estratégicas (habilidades para emplear la información contenida en los medios digitales como medio para alcanzar una meta personal o profesional en particular). Recientemente, Van Deursen y Van Dijk (2016) incluyen otras modalidades de habilidades como las de comunicación y las de creación de contenidos.

Las habilidades que se emplean para la comunicación y la creación de contenidos, así como aquellas relacionadas con la seguridad y privacidad, son fundamentales para crear una cultura digital, aunque no suficientes por sí mismas. Los trabajos de Jenkins, Clinton, Purushotma, Robison y Weigel (2006, p. 3) plantean el concepto de cultura participativa como una cultura con relativamente pocas barreras a la expresión artística y al compromiso cívico, con un apoyo importante para crear y compartir con los demás las propias creaciones y con un cierto tipo de enseñanza informal donde lo que es conocido por las personas más expertas pasa a las más noveles. Una cultura participativa es también aquella en la que sus miembros consideran importantes sus contribuciones y sienten cierto grado de conexión social entre ellos. El mismo acto de participar supone un grado de responsabilidad con respecto al conocimiento, compartir experiencias e informaciones con otros/as supone un papel activo en la generación y en el consumo de conocimiento. Estas habilidades marcan nuevas brechas digitales, en tanto que permiten a quienes las ha adquirido un mayor aprovechamiento de la inteligencia colectiva. No obstante, Schradie (2013) señala que la creación de contenidos y la participación no son procesos idénticos: mientras que la creación de contenidos potencialmente se dirige a un amplio público y puede ser unidireccional, la participación está comúnmente más centrada en grupos de referencia específicos y es de naturaleza más social e interactiva.

En este sentido, Internet y las redes sociales se convierten en una herramienta clave para la promoción de negocios y estimulan la actividad emprendedora. A través de Internet se logra una mayor difusión de productos y servicios y se llega a clientes potenciales. Esto permite a las mujeres compartir sus creaciones con los demás y dar difusión a sus trabajos. No obstante, contar con esta posibilidad no significa disponer de habilidades para hacerlo. De ahí, que nos interesemos por explorar los diferentes tipos de habilidades en Internet de las mujeres estudiantes e indagar en cómo influyen en su inclusión digital. 
El trabajo tiene como objetivos: a) describir las habilidades de las mujeres estudiantes en Internet, b) conocer el grado de inclusión digital de las mujeres estudiantes, c) establecer perfiles de mujeres en función a tipología y dominio de las habilidades en Internet, conociendo si influyen estos perfiles en una de las dimensiones clave de la inclusión digital, la confianza digital y d) analizar la relación de estos perfiles con el grado de inclusión digital global.

\section{Método}

\subsection{Participantes}

La muestra está compuesta por 215 mujeres estudiantes. El 68.4\% reside en Andalucía y el 31.6\% en Extremadura. El 93.9\% de las mujeres tiene una edad comprendida entre los 20 y 25 años, el 5.6\% tiene entre 26 y 34 años. Para su selección se ha seguido un muestreo no probabilístico por cuotas en base a la variable formación reciente (últimos tres años) en Internet. El 52.6\% de las mujeres participantes no ha recibido formación reciente, frente al 47.4\%, que sí ha recibido. Se siguen como criterios de inclusión de las mujeres en la muestra: a) la experiencia en internet de al menos un año y b) el uso frecuente de internet. La tabla 1 recoge las diferentes vías por las que las mujeres estudiantes consideran que han adquirido las habilidades en Internet. Así, se observa que el $84.4 \%$ ha desarrollado

\begin{tabular}{|c|c|c|}
\hline \multirow{2}{*}{ Formación previa en Internet } & Sí & 47.4 \\
\hline & No & 52.6 \\
\hline \multirow{2}{*}{$\begin{array}{c}\text { Ha desarrollado habilidades en Internet a } \\
\text { través de la institución educativa formal } \\
\text { (colegio, instituto, universidad, centros de } \\
\text { educación de adultos, etc.) }\end{array}$} & Sí & 81.6 \\
\hline & No & 18.4 \\
\hline \multirow{2}{*}{$\begin{array}{l}\text { Ha desarrollado habilidades en Internet } \\
\text { a través de cursos de formación que han } \\
\text { realizado por iniciativa propia }\end{array}$} & Sí & 26.3 \\
\hline & No & 73.7 \\
\hline \multirow{2}{*}{$\begin{array}{l}\text { Ha desarrollado habilidades en Internet } \\
\text { de forma autodidacta con libros, CD-ROM, } \\
\text { páginas web, blogs, tutoriales, YouTube, etc. }\end{array}$} & Sí & 58.6 \\
\hline & No & 41.4 \\
\hline \multirow{2}{*}{$\begin{array}{l}\text { Ha desarrollado habilidades en Internet de } \\
\text { forma autodidacta practicando (aprender } \\
\text { haciendo) }\end{array}$} & Sí & 83.6 \\
\hline & No & 16.4 \\
\hline \multirow{2}{*}{$\begin{array}{c}\text { Ha desarrollado habilidades en Internet a } \\
\text { través de ayuda informal de colegas, parientes, } \\
\text { amigos, hijos/as, etc. }\end{array}$} & Sí & 84.4 \\
\hline & No & 15.6 \\
\hline
\end{tabular}

Tabla 1. Formas de aprendizaje de las habilidades en Internet de las mujeres estudiantes. 
habilidades en Internet de manera informal a través de ayuda de colegas, parientes, amigos/as, hijos/ as, etc. El 83.6\% ha desarrollado habilidades en Internet de forma autodidacta practicando (aprender haciendo). Un bajo porcentaje de mujeres las desarrolla realizando cursos de formación (solo el $26.3 \%)$.

La recogida de datos se realiza a través de un cuestionario (online) que contiene variables sociodemográficas como la edad, formación previa en Internet, formas de desarrollo de las habilidades en Internet, así como dos escalas:

- Escala sobre alfabetización y competencia digital (habilidades en Internet). Esta escala tipo Likert con 18 ítems, en los que las estudiantes marcan la frecuencia con la que realizan una serie de actividades, presenta opciones que oscilan desde nunca, valor 0, a siempre, valor 3. Esta escala se diseña al efecto basándonos en las medidas propuestas por van Deursen, Helsper y Eynon (2014) y en informes técnicos del gobierno de Reino Unido. La fiabilidad de la escala se obtiene a partir del cálculo del alfa de Cronbach, obteniendo un alto coeficiente de .871.

- Escala sobre inclusión digital. Esta escala se compone de 12 ítems tipo Likert con valores que oscilan entre 0 (nunca) a 3 (siempre). La escala se diseña al efecto y se basa en aportaciones de Bradbrook y Fisher (2004) y Helsper (2008). La escala incluye ítems que miden la conectividad (por ejemplo, "tengo una conexión de banda ancha que me permite descargar con facilidad contenidos multimedia (vídeos, imágenes...)" o "accedo a Internet en cualquier lugar (casa, autobús, biblioteca,...)", la confianza digital (que incorpora ítems del tipo: "antes de realizar gestiones administrativas por Internet, compruebo el nivel de seguridad de la página") y la acción digital a través de la creación y el intercambio de contenidos culturalmente relevantes. Para la medida de la acción digital se usan ítems como "localizo contenidos de interés sobre mi localidad" o "accedo a información cultural relevante para mí". La fiabilidad de la escala se obtiene a partir del cálculo del alfa de Cronbach, obteniendo un alto coeficiente de .829.

\subsection{Procedimiento}

El cuestionario incorpora en su versión online, (puesta a disposición a través de la aplicación de Google Form), un consentimiento informado que requiere aceptación por parte de las mujeres antes de proceder a su cumplimentación. La recogida de información se realiza bajo una declaración de confidencialidad por parte de las personas que coordinan el estudio y se realiza entre los meses de abril y junio de 2015 .

El análisis de los datos se realiza mediante el paquete estadístico SPSS v. 23., aplicando técnicas exploratorias de las variables para conocer las características de su distribución y pruebas de validez 
y fiabilidad de las medidas empleadas. El procedimiento de análisis se basa en un análisis descriptivo de cada uno de los ítems de las escalas y un análisis exploratorio de las variables para conocer su estructura y distribución.

Para explicar los tipos de habilidades en Internet de las mujeres estudiantes, aplicamos un análisis factorial de componentes principales con rotación varimax a la medida de las habilidades en Internet determinando cinco factores que explican el $62.07 \%$ de la varianza total $32.03 \%$ el factor $1 ; 9.14 \%$ el factor 2; 7.35\% el factor 3; 7.22\% el factor 4 y 6.31 el factor 5). El análisis de la fiabilidad indica un alpha de Cronbach .776 para el factor 1 (habilidades en Internet para la e-administración), .73 para el factor 2 (habilidades en Internet para crear contenidos y compartirlos en redes sociales), .768 para el factor 3 (habilidades en Internet para el aprendizaje autónomo), .688 para el factor 4 (habilidades en Internet relacionadas con la privacidad y la seguridad) y .517 para el factor 5 (habilidades en Internet para la localización de información y la comunicación). Se observa en la tabla 2 que todos los ítems obtienen pesos factoriales superiores a .50, solo uno está muy próximo (con un valor de .46 y que hace referencia a "marco como favoritos los sitios web y servicios de interés"). Cada ítem se asigna al factor para el cuál su valor es más elevado.

\begin{tabular}{|c|c|c|c|c|c|c|}
\hline \multirow{2}{*}{\multicolumn{2}{|c|}{ HABILIDADES EN INTERNET }} & \multicolumn{5}{|c|}{ FACTORES } \\
\hline & & 1 & 2 & 3 & 4 & 5 \\
\hline \multirow{5}{*}{ 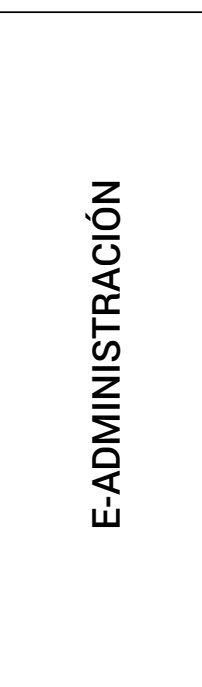 } & $\begin{array}{l}\text { Administro y consulto mi } \\
\text { cuenta bancaria }\end{array}$ & .791 & -.034 & .207 & .112 & -.015 \\
\hline & $\begin{array}{c}\text { Realizo compras online de } \\
\text { productos y servicios (viajes, } \\
\text { hoteles, ropa, libros, teatros, } \\
\text { cine, etc.) }\end{array}$ & .769 & .131 & .111 & -.026 & -.025 \\
\hline & $\begin{array}{c}\text { Realizo gestiones } \\
\text { administrativas por Internet } \\
\text { (estudios, salud, etc.) }\end{array}$ & .661 & .092 & .157 & .227 & .400 \\
\hline & $\begin{array}{c}\text { Me comunico por Internet para } \\
\text { preguntar por productos y } \\
\text { servicios }\end{array}$ & .578 & .391 & .180 & .028 & 230 \\
\hline & $\begin{array}{l}\text { Marco como favoritos los sitios } \\
\text { web y servicios que veo útiles }\end{array}$ & .461 & .099 & .204 & .283 & 325 \\
\hline \multirow{4}{*}{ 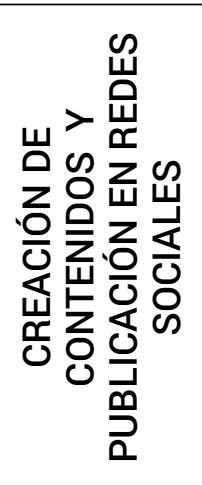 } & $\begin{array}{l}\text { Publico contenidos originales } \\
\text { propios en Internet }\end{array}$ & .138 & .777 & .217 & .216 & .039 \\
\hline & $\begin{array}{l}\text { Creo y comparto fotos y/o } \\
\text { vídeos por Internet }\end{array}$ & .036 & .750 & .125 & .210 & 211 \\
\hline & $\begin{array}{l}\text { Creo y mantengo webs, blogs } \\
\text { y/o canales de YouTube propios } \\
\text { sobre temas de mi interés }\end{array}$ & .165 & .642 & .233 & .110 & -.156 \\
\hline & $\begin{array}{c}\text { Participo en foros y redes } \\
\text { sociales para comunicarme y } \\
\text { estar informada }\end{array}$ & .011 & .563 & .054 & -.146 & .535 \\
\hline
\end{tabular}




\begin{tabular}{|c|c|c|c|c|c|c|}
\hline \multirow{4}{*}{ 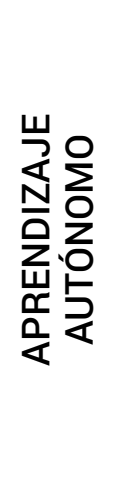 } & $\begin{array}{l}\text { Aprendo a resolver tareas } \\
\text { usando tutoriales de Internet }\end{array}$ & .123 & .169 & .824 & .068 & .136 \\
\hline & $\begin{array}{c}\text { Utilizo los comentarios de } \\
\text { otras personas en Internet para } \\
\text { resolver dudas }\end{array}$ & .091 & .146 & .796 & .083 & .213 \\
\hline & $\begin{array}{c}\text { Acudo a servicios de ayuda } \\
\text { técnica para resolver problemas }\end{array}$ & .344 & .193 & .621 & -.008 & .092 \\
\hline & $\begin{array}{c}\text { Realizo copias periódicas de } \\
\text { seguridad en dispositivos } \\
\text { externos }\end{array}$ & .194 & .109 & .592 & .263 & .042 \\
\hline \multirow{3}{*}{ 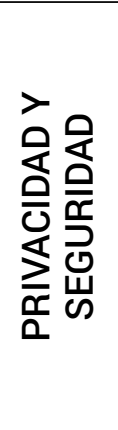 } & $\begin{array}{c}\text { Configuro las opciones de } \\
\text { privacidad para proteger mis } \\
\text { datos personales }\end{array}$ & -.068 & -.023 & .187 & .749 & .193 \\
\hline & $\begin{array}{c}\text { Me descargo e instalo } \\
\text { programas de sitios web } \\
\text { seguros }\end{array}$ & .276 & .285 & .091 & .689 & .031 \\
\hline & $\begin{array}{l}\text { Comparto contenidos en } \\
\text { Internet respetando la } \\
\text { propiedad intelectual }\end{array}$ & .162 & .434 & .023 & .651 & .060 \\
\hline \multirow{2}{*}{ 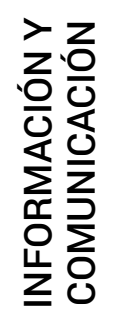 } & $\begin{array}{l}\text { Utilizo Google (u otro motor de } \\
\text { búsqueda) para encontrar la } \\
\text { información que necesito }\end{array}$ & .042 & -.043 & .099 & .266 & .704 \\
\hline & $\begin{array}{c}\text { Uso correo electrónico, } \\
\text { videollamadas y mensajería } \\
\text { instantánea para comunicarme } \\
\text { por Internet }\end{array}$ & .237 & .127 & .276 & .036 & .698 \\
\hline
\end{tabular}

Para la medida de la inclusión digital y siguiendo el mismo procedimiento analítico explicado con anterioridad, se obtienen cuatro factores que explican el $62.47 \%$ de la varianza total $(29.244 \%$ el factor 1, $13.079 \%$, el factor $2 ; 11.541 \%$, el factor 3 y $8.603 \%$, el factor 4 ). La tabla 3 muestra los ítems de la escala de inclusión digital y sus pesos factoriales.

\begin{tabular}{|c|c|c|c|c|c|}
\hline & \multirow{2}{*}{ INCLUSIÓN DIGITAL } & \multicolumn{4}{|c|}{ FACTORES } \\
\hline & & 1 & 2 & 3 & 4 \\
\hline \multirow{3}{*}{ 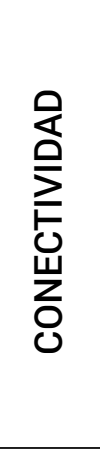 } & $\begin{array}{l}\text { Accedo a Internet en cualquier lugar } \\
\text { (casa, autobús, biblioteca, etc.) }\end{array}$ & .138 & .111 & .804 & .031 \\
\hline & $\begin{array}{l}\text { Utilizo dispositivos móviles propios } \\
\text { (que solo uso yo) para acceder a } \\
\text { Internet }\end{array}$ & .031 & .037 & .829 & .053 \\
\hline & $\begin{array}{c}\text { Tengo una conexión de banda ancha } \\
\text { que me permite descargar con facilidad } \\
\text { contenidos multimedia (vídeos, } \\
\text { imágenes,etc.) }\end{array}$ & .155 & .165 & 637 & -.182 \\
\hline 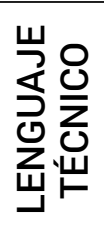 & $\begin{array}{l}\text { Tengo dificultades para entender } \\
\text { lenguajes que aparecen cuando navego } \\
\text { por Internet (pluggins, cookies, etc.) }\end{array}$ & -.042 & -.075 & -.053 & .902 \\
\hline
\end{tabular}




\begin{tabular}{|c|c|c|c|c|c|}
\hline \multirow{4}{*}{ 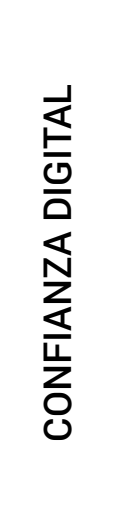 } & $\begin{array}{l}\text { Reconozco cuando entro en sitios web } \\
\text { que son inseguros }\end{array}$ & .139 & .451 & .019 & .352 \\
\hline & $\begin{array}{l}\text { Configuro opciones en los programas } \\
\text { que utilizo de internet para que mi } \\
\text { información personal esté segura }\end{array}$ & .178 & .717 & .049 & -.106 \\
\hline & \begin{tabular}{|c|} 
Antes de realizar gestiones \\
administrativas por Internet compruebo \\
el nivel de seguridad de la página
\end{tabular} & .121 & .789 & .192 & -.095 \\
\hline & $\begin{array}{l}\text { Me aseguro de que el entorno sea } \\
\text { seguro y confiable para publicar } \\
\text { información personal en Internet }\end{array}$ & -.032 & .815 & .119 & .059 \\
\hline \multirow{4}{*}{ 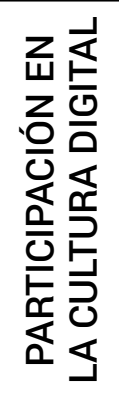 } & $\begin{array}{l}\text { Encuentro contenidos comprensibles } \\
\text { para mi nivel formativo }\end{array}$ & .589 & .227 & .361 & .123 \\
\hline & $\begin{array}{c}\text { Accedo a información cultural relevante } \\
\text { para mí }\end{array}$ & .728 & .196 & .308 & .053 \\
\hline & $\begin{array}{c}\text { Localizo contenidos de interés sobre mi } \\
\text { localidad }\end{array}$ & .857 & .075 & .031 & .036 \\
\hline & $\begin{array}{l}\text { Publico información interesante sobre } \\
\text { mi localidad }\end{array}$ & .751 & .001 & -.024 & -.133 \\
\hline
\end{tabular}

Tabla 3. Matriz de componentes rotados para la escala de inclusión digital.

En este trabajo nos interesa especialmente medir el grado de inclusión digital global. Para ello, comprobamos la unidimensionalidad de la escala de inclusión digital a través de un análisis de componentes principales para datos categóricos. Los resultados de este análisis permiten crear una variable a partir del sumatorio de los ítems de la escala de inclusión digital quedando la nueva variable en una escala de medida con valores que oscilan entre 0 y 36 puntos. A partir de este procedimiento se recodifica la variable en tres nuevas modalidades: 1) Nivel bajo de inclusión digital (puntuación de 0 a 12); Nivel medio de inclusión digital (puntuación de 13 a 24) y nivel elevado de inclusión digital (puntuación de 25 a 36). Esta recodificación no afecta a su validez y fiabilidad.

Posteriormente, realizamos un análisis de conglomerados de K medias (Q-Cluster) para establecer perfiles de mujeres estudiantes en función de los tipos de habilidades en Internet y su nivel de dominio. Una vez aplicada la prueba de Kolmogorov-Smirnov con la que se rechaza la hipótesis de normalidad de las distribuciones, se aplica la prueba de contraste no paramétrica de Kruskal-Wallis para muestras independientes con el objeto de estudiar si existen diferencias estadísticamente significativas en la confianza digital de los diferentes perfiles de mujeres. Por último, se aplican tablas de comparación cruzada entre los perfiles de las mujeres estudiantes según sus habilidades y el grado de inclusión digital. Para el estudio de la relación entre ambas variables se usa el coeficiente de contingencia.

\section{Resultados}

A continuación presentamos los resultados de investigación de acuerdo a los objetivos. 


\subsection{Habilidades en Internet de las mujeres estudiantes}

Las habilidades en Internetrelacionadas con la realización de gestiones administrativas ytransacciones comerciales, muestra que el $44.1 \%$ de las mujeres estudiantes marcan como favoritos los servicios que ven útiles, seguido de un 37.1\% que realiza con frecuencia gestiones administrativas por Internet y de un $34.6 \%$ de mujeres que solo algunas veces realizan compras online de productos y servicios (viajes, libros, teatro,...). Esto supone que el 68.6\% de mujeres hacen uso de la e-administración y se manejan en este sentido. Estos datos contrastan con el 39.3\% de las mujeres que nunca administran y consultan cuentas bancarias.

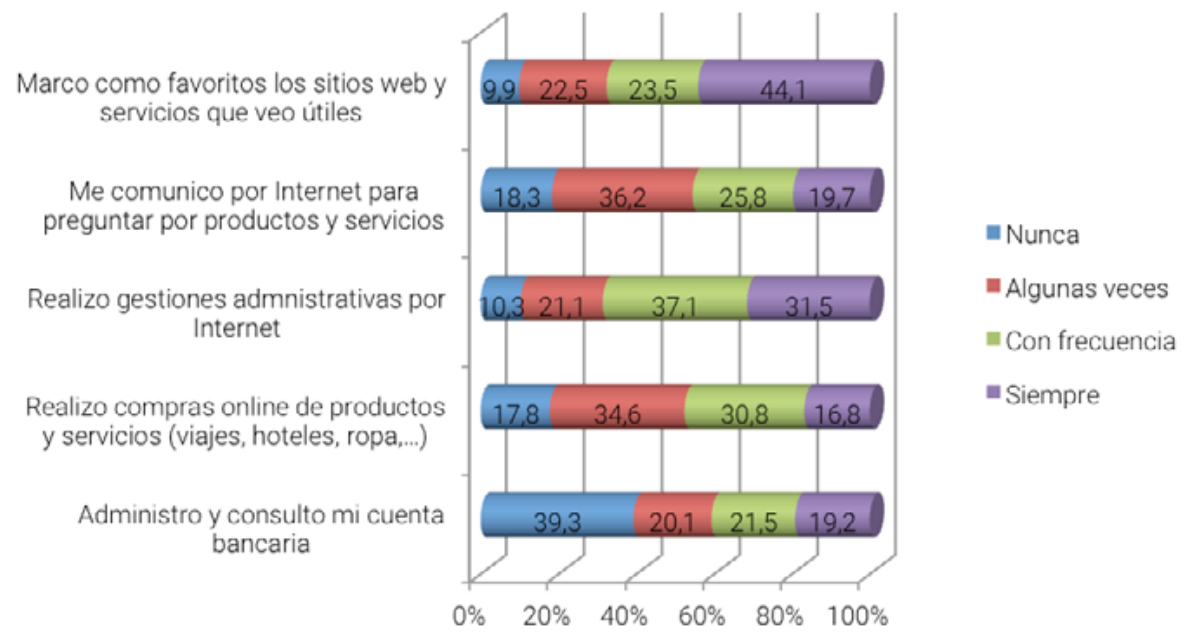

Gráfico 1. Habilidades para la e-administración.

Con respecto a las habilidades para crear y compartir contenidos en Internet tales como fotografías, vídeos, noticias, etc., un 55.9\% de las mujeres nunca han creado y desarrollado webs, blogs o canales de YouTube sobre temas de su propio interés. A esto hay que añadir que el $35.2 \%$ nunca publica contenidos originales propios en Internet. Sin embargo, sí muestran una gran actividad en lo que respecta a crear y compartir fotos con frecuencia (41.3\%) y participar en foros y redes sociales (31.8\%).

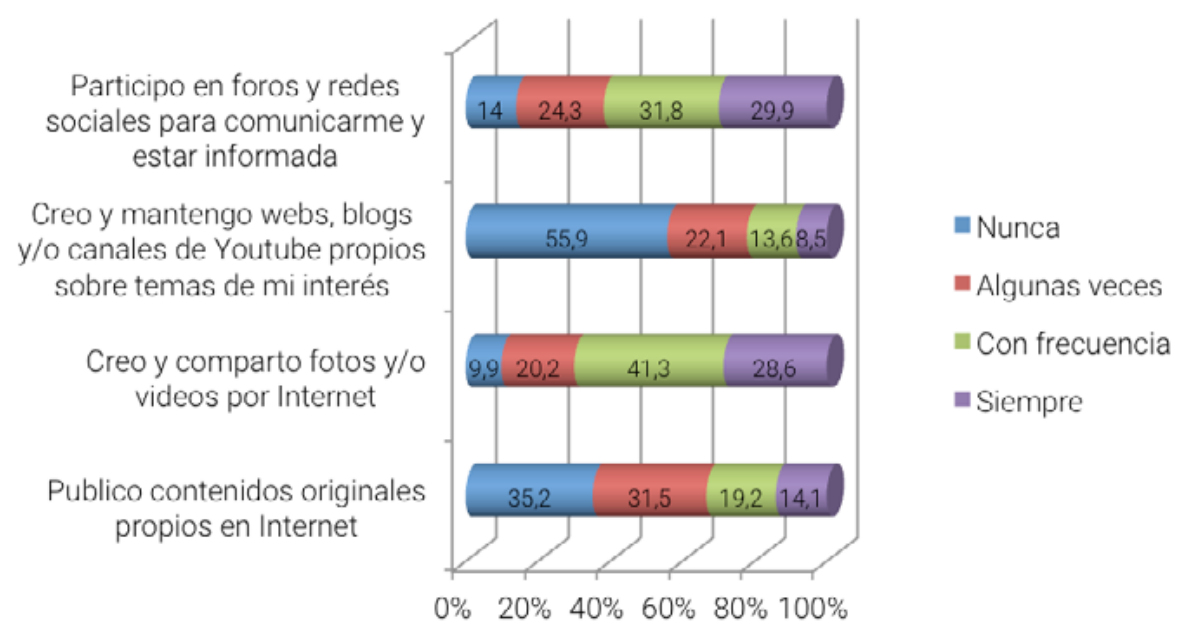


En relación a las habilidades en Internet para el aprendizaje autónomo, un notable porcentaje de mujeres (46\%) recurre algunas veces a servicios de ayuda técnica para aprender a resolver problemas, seguido de un $37.3 \%$ y un $36.4 \%$ respectivamente, que se sirve de comentarios de otras personas y tutoriales para resolver sus dudas y tareas. En relación a las habilidades relacionadas con el aprendizaje a través de la red, se observa que un $27.1 \%$, nunca realiza copias periódicas de seguridad en dispositivos externos. Solo el 18.1\% reconoce hacer copias periódicas siempre.

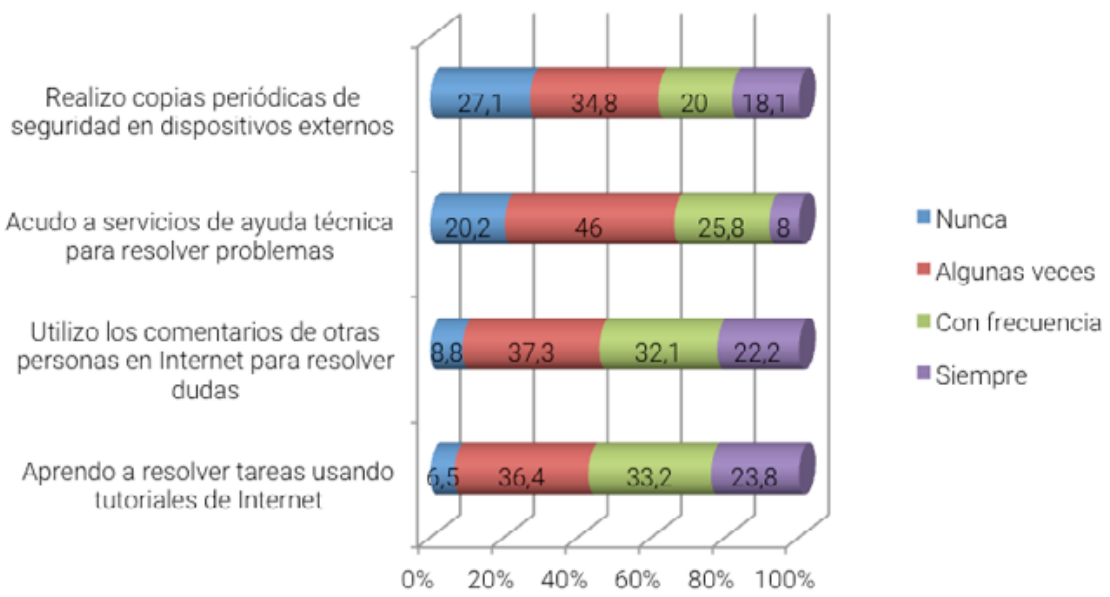

Gráfico 3. Habilidades para el aprendizaje autónomo.

En cuanto a las habilidades relacionadas con la privacidad y la seguridad un alto porcentaje (71.3\%) configura siempre o con frecuencia las opciones de privacidad para proteger sus datos personales. En contraposición, un 31.5\% indica que solo algunas veces comparte contenidos en Internet respetando la propiedad intelectual.

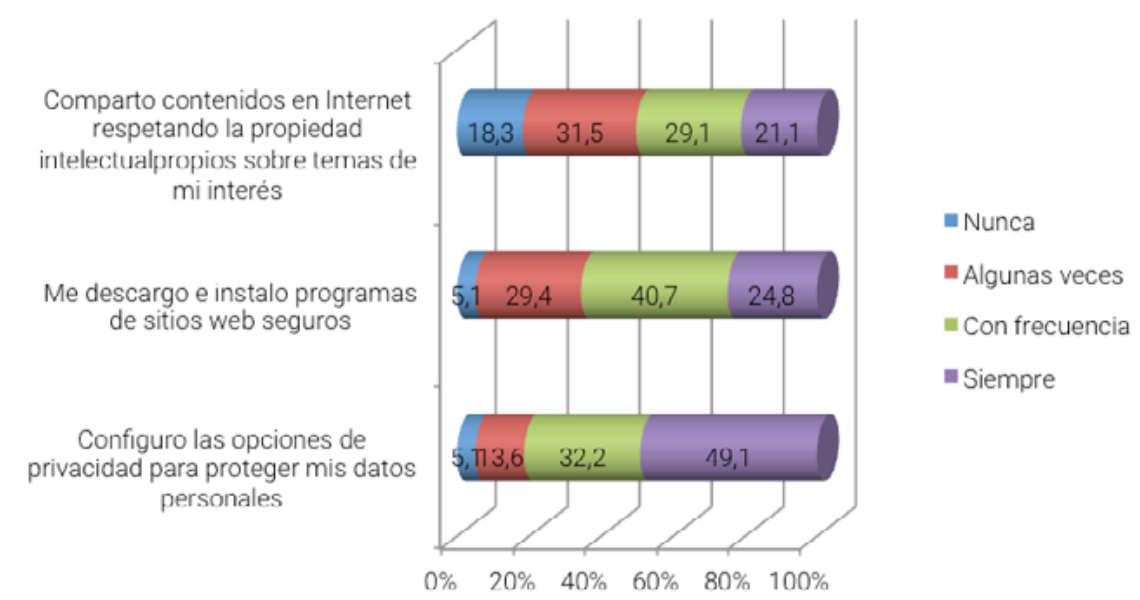

Gráfico 4. Habilidades relacionadas con la privacidad y la seguridad en Internet.

Por último, por lo que respecta a las habilidades básicas para la localización de información y la comunicación, los datos muestran que un notable 79.9\% de mujeres hacen uso de Google para localizar información. Resulta llamativo que un $45.4 \%$ de mujeres solo algunas veces usen una diversidad de herramientas (correo electrónico, videollamadas, etc.) para comunicarse por Internet. 


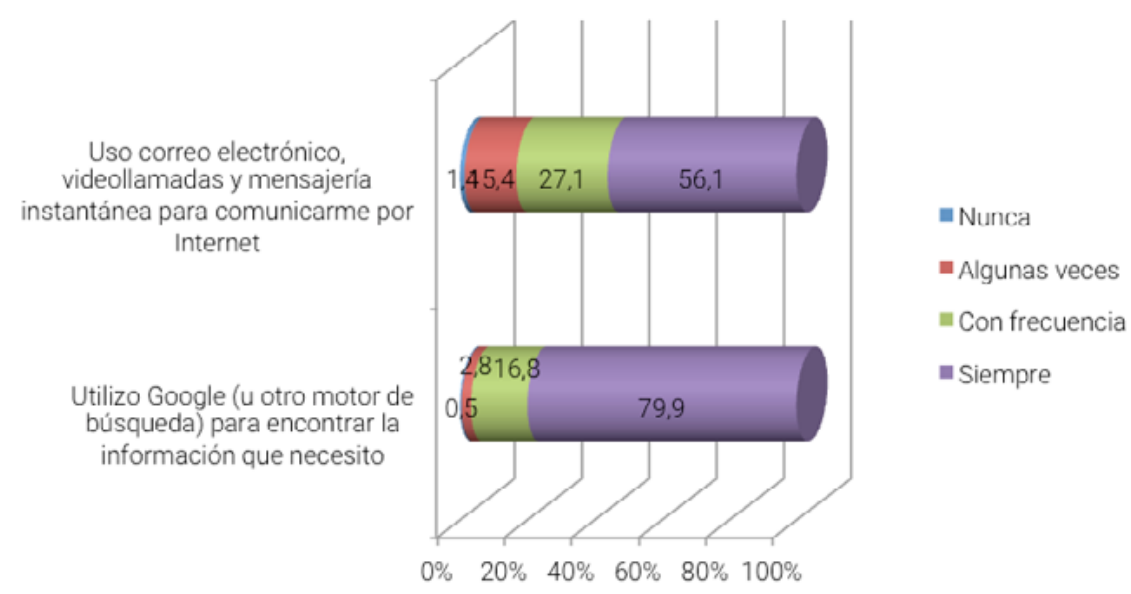

Gráfico 5. Habilidades para la localización de información y comunicación por Internet.

\subsection{Grado de inclusión digital de las mujeres estudiantes}

Las mujeres que participan en el estudio muestran un nivel medio de inclusión digital. Así, el 61.4\% de las mujeres muestran un nivel medio de inclusión digital, un 36.2\% muestran un grado elevado de inclusión y solo un 2.4\% muestra un grado bajo de inclusión.

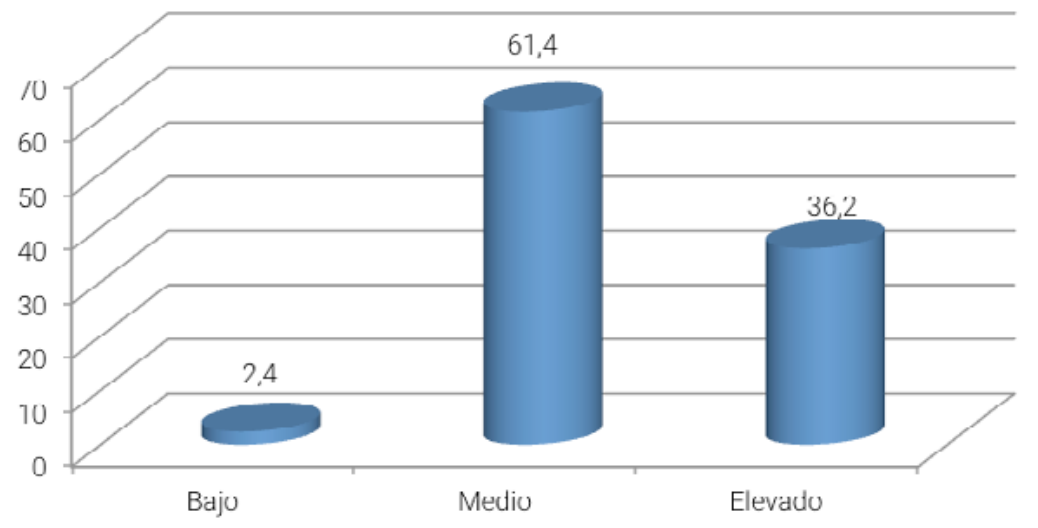

Gráfico 6. Grados de inclusión digital de las mujeres estudiantes.

En lo que respecta a la inclusión digital, se observa que la dimensión de conectividad es la que alcanza un mayor valor de la media $(M=2.7)$. Así, la dimensión que hace referencia al lenguaje técnico es la que tiene una menor media $(M=1.3)$. Esta dimensión hace referencia al conocimiento del lenguaje técnico característico de la actividad en Internet (pluggins, cookies, etc.).

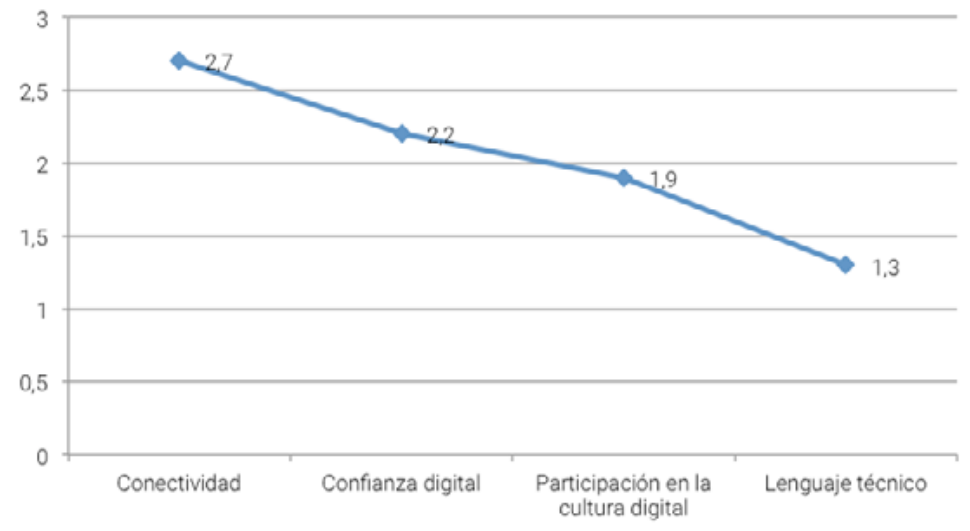

Gráfico 7. Media de las dimensiones de la inclusión digital. 
En este sentido, el Gráfico 8 muestra que el 75.8\% de las mujeres disfrutan de un grado alto de conectividad. Sin embargo, en lo que respecta al uso y manejo de un lenguaje técnico, el $70.2 \%$ de las mujeres muestran dificultades. Solo un $7.9 \%$ de las estudiantes muestra un alto nivel en esta dimensión.

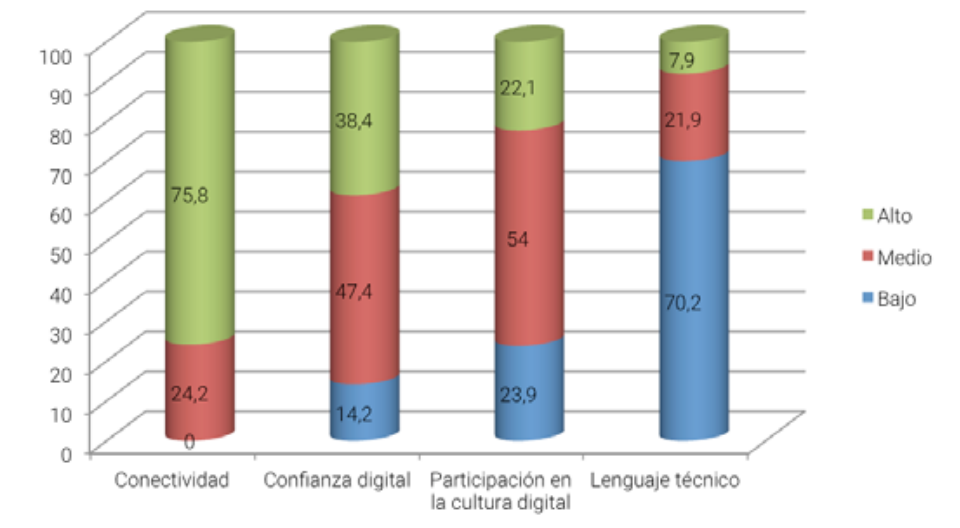

Gráfico 8. Grado de inclusión digital de las mujeres estudiantes por dimensiones

\subsection{Perfiles de mujeres estudiantes según sus habilidades en Internet y relación con la inclusión digital}

Estudios previos (Jiménez, Vega \& Vico, 2015) ponían de manifiesto que existe relación entre la competencia digital y la inclusión digital. El estudio al que hacemos referencia muestra que la variable competencia digital explica el $24.5 \%$ de la inclusión digital. Concretamente, en el estudio previo mencionado el análisis de cada uno de los grupos de habilidades en Internet muestra que las habilidades relacionadas con la seguridad y privacidad en Internet explican un $29.3 \%$ de la inclusión digital. Concretamente, en este trabajo que presentamos ahora, nos interesa profundizar en este resultado y conocer qué tipos de habilidades en Internet caracterizan a las mujeres que tienen un mayor nivel de inclusión digital. Para ello, aplicamos un análisis de clúster identificando conjuntos de habilidades que caracterizan a diferentes grupos de mujeres estudiantes.

Los resultados muestran cuatro perfiles de mujeres estudiantes en función de los tipos de habilidades en Internet y su nivel de dominio: a) grupo 1 principiante "beginner" (28.6\%), que aglutina a mujeres que muestran una gran variedad de habilidades sin desarrollarlas en su totalidad, es decir, no llegan a perfeccionarlas; b) grupo 2 intermedio "average" (41.4\%), este grupo muestra un nivel medio en el manejo de este tipo de destrezas; c) grupo 3 avanzado "advanced" (25.6\%) que indica que sus mayores habilidades se concentran en tres aspectos fundamentales, relacionados con la gestión administrativa, creación de contenidos en redes sociales y aprendizaje autónomo; y d) grupo 4 especializado en gestión digital (4.4\%), con habilidades muy centradas en la e-administración. 


\begin{tabular}{|c|c|c|c|c|c|c|c|c|c|c|}
\hline \multirow{3}{*}{$\begin{array}{l}\text { Factores de } \\
\text { Habilidades en } \\
\text { Internet }\end{array}$} & \multicolumn{4}{|c|}{$\begin{array}{c}\text { Centros de } \\
\text { conglomerados } \\
\text { finales } \\
\text { (4 grupos) }\end{array}$} & \multicolumn{6}{|c|}{ ANOVA } \\
\hline & & & & & \multicolumn{2}{|c|}{ Conglomerado } & \multicolumn{2}{|c|}{ Error } & \multirow[b]{2}{*}{$\mathbf{F}$} & \multirow[b]{2}{*}{ Sig. } \\
\hline & 1 & 2 & 3 & 4 & $\begin{array}{l}\text { Media } \\
\text { cuadrática }\end{array}$ & GI & $\begin{array}{c}\text { Media } \\
\text { cuadrática }\end{array}$ & GI & & \\
\hline E-Administración & 4 & 8 & 12 & 11 & 704,999 & 3 & 3,838 & 199 & 183,711 &, 000 \\
\hline $\begin{array}{c}\text { Creación de } \\
\text { contenidos y } \\
\text { comparto en redes } \\
\text { sociales }\end{array}$ & 4 & 5 & 9 & 2 & 263,441 & 3 & 5,133 & 199 & 51,320 &, 000 \\
\hline $\begin{array}{l}\text { Aprendizaje } \\
\text { autónomo }\end{array}$ & 4 & 6 & 9 & 2 & 286,051 & 3 & 4,084 & 199 & 70,041 & ,000 \\
\hline $\begin{array}{l}\text { Privacidad y } \\
\text { seguridad }\end{array}$ & 4 & 5 & 7 & 7 & 88,834 & 3 & 3,306 & 199 & 26,868 &, 000 \\
\hline $\begin{array}{l}\text { Información y } \\
\text { comunicación } \\
\text { básica }\end{array}$ & 4 & 5 & 6 & 4 & 23,308 & 3 & 876 & 199 & 26,604 &, 000 \\
\hline
\end{tabular}

Desde un punto de vista exploratorio, el gráfico 9 muestra las diferencias en la inclusión digital en función de los cuatro perfiles de mujeres según los tipos de habilidades en Internet. El grupo "avanzado" es el que cuenta con un mayor grado de inclusión digital $(M=26.59 ; D T=3.91)$ seguido del grupo "especializado en e-administración" ( $M=23 ; \mathrm{DT}=3.16)$. Esto pone de manifiesto la repercusión que puede tener este tipo de habilidades tan específicas para la inclusión digital.

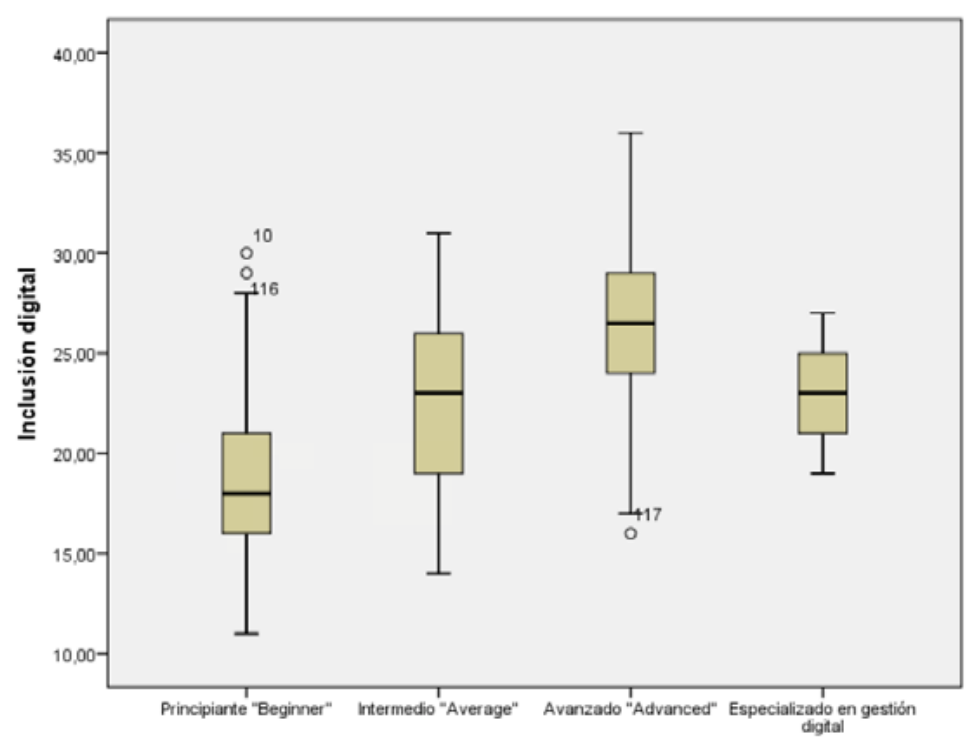

Gráfico 9. Inclusión digital de los diferentes perfiles de mujeres.

No obstante, el estudio de algunas dimensiones claves de la inclusión digital por separado, nos permite aportar hallazgos sobre la relevancia del tipo de habilidades implicadas en el uso de Internet. Por ello, 
analizamos, por un lado, la confianza digital en los diferentes perfiles de mujeres estudiantes (Gráfico 10) y por otro lado, la participación en la cultura digital, es decir, a través de la creación de contenidos culturalmente relevantes (Gráfico 11).

En el Gráfico 10 podemos observar que, en la dimensión de confianza digital, las medias de los grupos "avanzado" ( $M=8.75 ; \mathrm{DT}=2.21$ ) y del especializado en gestión digital son similares ( $M=8.33$; $\mathrm{DT}=2.69)$. Esto indica que las habilidades relacionadas con la gestión, la administración digital y el comercio, junto con las habilidades para garantizar la privacidad y la seguridad son suficientes en sí mismas para alcanzar la confianza digital de las mujeres estudiantes contribuyendo, en este sentido, a elevar su grado de inclusión digital. La prueba de Kruskal Wallis=25.807, gl=3, p=.000) indica que estas diferencias son estadísticamente significativas.

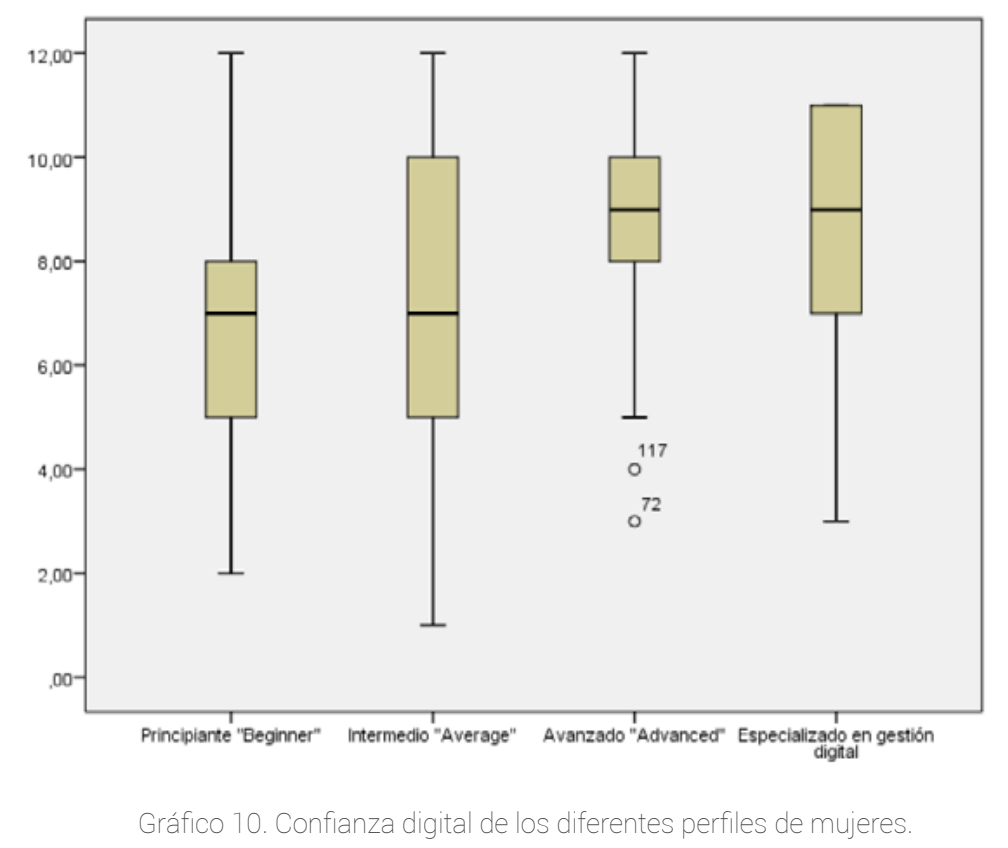

No obstante, si estudiamos otra dimensión de la inclusión digital como es la participación en la cultura digital de las mujeres estudiantes (ver Gráfico 11), las habilidades en gestión digital no contribuyen por sí solas a elevar la inclusión digital. El contenido relevante es aquel que por su valor cultural, por resultar contenido representativo o importante de sus localidades, etc., es compartido y/o creado por ellas.

Se observa también que el grupo con habilidades avanzadas es el que alcanza una mayor media en esta dimensión de la inclusión digital ( $M=8.50$; DT=2.35) en comparación, por ejemplo, con el grupo que hemos denominado "especializado en e-administración" ( $M=5.88$; DT=1.45). El grupo "avanzado" cuenta con habilidades concretas como crear contenidos, mantener canales en YouTube, compartir fotos y eventos en redes sociales, además de que también cuenta con las habilidades para la gestión digital. En este sentido, el desarrollo de las habilidades relacionadas con la creación y el intercambio de contenidos relevantes para las mujeres estudiantes repercuten en esta dimensión de la inclusión digital contribuyendo así, esta última, a la inclusión social. 


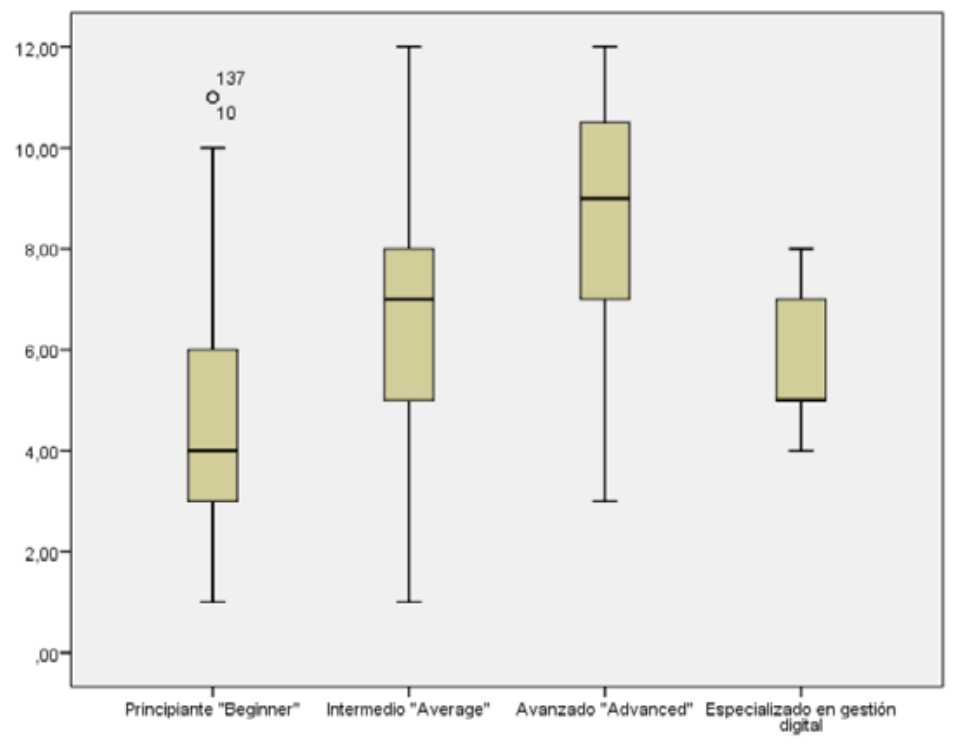

Gráfico 11. Participación en la cultura digital de los diferentes perfiles de mujeres.

Por último, en el gráfico 12, se puede observar que existe una marcada diferencia en el grado de inclusión digital entre el grupo "avanzado" (donde el 73.1\% muestra un grado elevado de inclusión digital) y el grupo "especializado en e-administración" donde un 44.4\% de las mujeres estudiantes muestran un elevado grado de inclusión digital. Lo que nos indica que no es la diversidad de habilidades en Internet por sí sola el aspecto que más se relaciona con un grado elevado de inclusión digital, sino la diversidad y un dominio elevado.

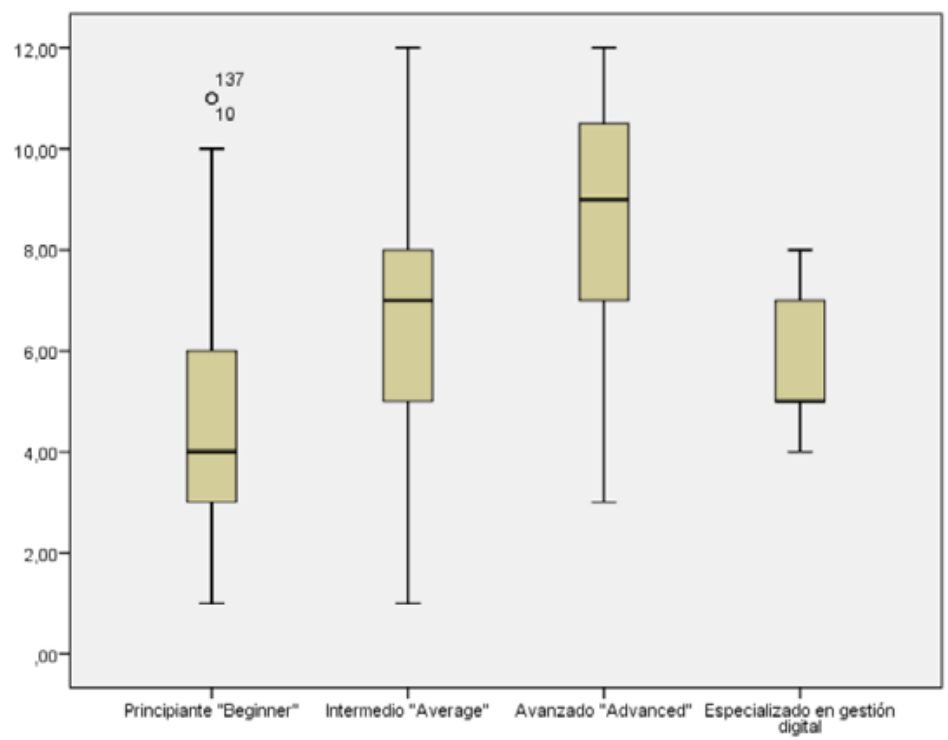

Gráfico 12. Perfiles de mujeres en función de las habilidades en Internet y grado de inclusión digital.

Así, se observa que el 8.8\% de las mujeres estudiantes del grupo "beginner" tienen un grado bajo de inclusión digital. No encontrándose, este grado bajo de inclusión digital en ninguno de los otros perfiles. El coeficiente de contingencia $\left(X^{2=62312, C=.488, p=.000}\right)$ muestra la existencia de diferencias en la inclusión digital en las mujeres estudiantes con diferente perfil de habilidades en Internet. 


\section{Discusión de resultados y conclusiones}

Este estudio está motivado por la necesidad de explorar diferentes tipos de habilidades en Internet y, especialmente, conocer las más relacionadas con un grado elevado de inclusión digital. Este conocimiento permite preparar a las mujeres para una ciudadanía digital activa y plena, ya que favorece priorizar tipos de habilidades en Internet a la hora de plantear propuestas formativas orientadas a distintos propósitos (empleabilidad, bienestar, compromiso cívico, desarrollo profesional,...). Este trabajo pone de manifiesto diferentes tipos de habilidades de las mujeres estudiantes a través de distintas actividades que realizan en Internet. De forma consistente con otros trabajos (Espinar y Rodríguez, 2009; Menéndez, 2012) aquellas habilidades más relacionadas con el uso de servicios avanzados de Internet como la realización de videollamadas o la creación y publicación de contenidos propios a través de las redes sociales no suelen ser puestas en práctica por un amplio porcentaje de mujeres. Así, informes técnicos del Instituto de la Mujer para la e-igualdad (Fundación Directa, 2011) muestran que las mujeres desarrollan en menor medida habilidades relacionadas con actividades de mayor especialización técnica, relacionadas con manejar programas, comprimir ficheros, conectar o instalar dispositivos o usar lenguajes de programación. En el mismo sentido, se observa en nuestro estudio que las mujeres estudiantes muestran dificultades en el lenguaje técnico relacionado con Internet, es decir, en el manejo de terminología especializada (pluggins, cookies, etc.). El lenguaje técnico aparece en este trabajo como un claro obstáculo a la inclusión digital de las mujeres estudiantes. Eszter Hargittai (2005) propuso medidas sensibles a esta realidad creando incluso preguntas para medir el conocimiento de términos de Internet en las encuestas (por ejemplo, búsqueda avanzada, jpg, pdf, spyware, malware). Según Menéndez (2012) estas brechas digitales ponen en riesgo el futuro profesional, social e incluso identitario de las mujeres en un mundo definido por las tecnologías digitales.

Los resultados del estudio que presentamos ponen de manifiesto una necesidad de orientar las habilidades de las estudiantes a la creación de contenidos digitales propios y su distribución en Internet. En este trabajo, hemos puesto de manifiesto que un destacado 55.9\% de las mujeres estudiantes no crea ni mantiene nunca webs, blogs y/o canales de YouTube sobre temas de interés, solo lo hace algunas veces el $22.1 \%$, por lo que son habilidades escasamente practicadas por un amplio número de mujeres y resultan claves en la configuración de una cultura participativa. Esto incide en las nuevas brechas digitales, concretamente la brecha de participación a la que aluden Hargittai y Walejko (2008) y Hoffmann, Lutz, y Meckel (2015). Y es que, el compromiso cívico parte de crear y compartir con los demás las propias creaciones y asumir una implicación con el apoyo a otras mujeres menos experimentadas. La cultura participativa requiere que las mujeres consideren importantes sus contribuciones y sientan conexión social entre ellas. El mismo acto de participar supone un grado de 
responsabilidad con respecto al conocimiento, compartir experiencias e informaciones con otros/as supone un papel activo en la generación y en el consumo de conocimiento, de ahí su relevancia. El paso de las mujeres de consumidoras a prosumidoras depende de este tipo de habilidades (Menéndez, 2012), así el estudio de Correa (2010), muestra que las mujeres son menos creadoras de contenido que los hombres.

Este estudio pone de manifiesto que un amplio porcentaje de mujeres estudiantes se manejan en la administración digital y en la realización de trámites a través de Internet, es muy probable que por la edad y la situación de dependencia económica de la familia, el 39.3\% nunca administra ni consulta su cuenta bancaria a través de Internet. Estos hallazgos son relevantes para el análisis de la inclusión digital de las mujeres estudiantes y del ejercicio de la ciudadanía digital. Estos resultados son coincidentes con las aportaciones de Collado, Martín y Vázquez (2008), ya que las mujeres usan las tecnologías digitales desde una perspectiva muy funcional y para hacer gestiones de la vida diaria relacionadas con la salud, los viajes, los trámites administrativos de matrículas y estudios, etc.

El estudio pone de manifiesto que las habilidades relacionadas con la privacidad son puestas en práctica con mayor frecuencia por las mujeres. No obstante, nuestros resultados coinciden con los de AlonsoRuido, Rodríguez-Castro, Lameiras-Fernández y Carrera-Fernández (2015), mostrando que las chicas son más cuidadosas en la privacidad de sus perfiles, teniendo en mayor medida perfiles cerrados/ protegidos. El estudio de estas autoras indica que las chicas declaran que solo sus amistades pueden ver sus perfiles y son ellas las que tienen mayor conciencia sobre el peligro de las redes sociales.

La literatura apunta a que las habilidades relacionadas con la privacidad en el espacio público en red pueden ser un aspecto que diferencie y caracterice la alfabetización digital en mujeres de diferente edad (Lara, 2011). Por lo que nos interesa describirla en diferentes edades, constituyendo este estudio un primer paso. Consideramos que es preciso profundizar en esta hipótesis ampliando la muestra a mujeres de diferentes edades y realizando una aproximación cualitativa a las formas de uso de internet en el marco de las diferentes actividades propuestas para poder contrastarla.

Desde un punto de vista metodológico, sabemos que los estudios internacionales ponen en cuestión la medida de las habilidades a través de técnicas de autoinforme (van Dijk, 2006), en este sentido, estamos desarrollando otro tipo de técnicas basadas en protocolos de pensamiento en voz alta (Greenhow y Robelia, 2009) para completar nuestros estudios sobre las habilidades en internet y aplicándolos a mujeres estudiantes. No obstante, creemos que este tipo de medidas son necesarias. Así el estudio de Correa (2010) pone de manifiesto que la autopercepción sobre las habilidades digitales influye en la creación de contenidos.

En resumen, destacamos tres conclusiones relevantes: 
- Las propuestas formativas que se planteen para la mejora de la inclusión digital de las mujeres han de tener en cuenta grupos de habilidades específicas. Así, las habilidades para la e-administración juegan un importante papel en el aumento de la inclusión digital y resultan claves en el uso de servicios digitales, principalmente porque contribuyen a aumentar la confianza digital de las mujeres. No obstante, estas habilidades no sirven por sí solas para aumentar la participación en la cultura digital.

- Prestar atención a nuevas brechas digitales, como las ocasionadas por una falta de habilidades relacionadas con la participación y la creación e intercambio de contenidos propios en Internet, requiere priorizarlas y capacitar a las mujeres en ellas. Estas habilidades, se relacionan con procesos de inclusión social localmente situados y culturalmente relevantes para ellas, así como con formas y estrategias avanzadas en el aprovechamiento de la inteligencia colectiva, especialmente, a través del uso de las redes sociales.

- Los lenguajes técnicos relacionados con la actividad en Internet suponen un obstáculo para la inclusión digital de las mujeres. El desarrollo de un lenguaje inclusivo y/o la capacitación en lenguajes de programación se presentan como aspectos prioritarios para superar la brecha digital de género.

\section{Referencias}

Alonso-Ruido, P., Rodríguez-Castro, Y., Lameiras-Fernández, M., y Carrera-Fernández, M. (2015). Hábitos de uso en las Redes Sociales de los y las adolescentes: análisis de género. Revista de Estudios e Investigación en Psicología y Educación, 0(13), 054-057. doi: http://dx.doi.org/10.17979/ reipe.2015.0.13.317

Area-Moreira, M., y Ribeiro-Pessoa, T. (2012). De lo sólido a lo líquido: Las nuevas alfabetizaciones ante los cambios culturales de la Web 2.0. Comunicar, XIX, 38, 13-20. doi: http://dx.doi.org/10.3916/C382012-02-01

Aviram, A., y Eshet-Alkalai, Y. (2006). Towards a theory of digital literacy: three scenarios for the next steps. European Journal of Open, Distance and E-Learning, 1, 1-11.

Bradbrook, G., y Fisher, J. (2004). Digital Equality: Reviewing Digital Inclusion Activity and Mapping the Way. Forwards. London: Citizens Online.

Castaño, C., Martín, J. y Vázquez, S. (2008). La e-inclusión y el bienestar social: una perspectiva de género. Economía Industrial, 367, 139-152. 
Correa, T. (2010). The participation divide among "online experts": Experience, skills and psychological factors as predictors of college students' web content creation. Journal of Computer Mediated Communication,16(1), 71-92. doi: http://dx.doi.org/10.1111/j.1083-6101.2010.01532.x

Correa, T., y Jeong, S.H. (2011). Race and online content creation: Why minorities are actively participating in the Web. Information, Communication \& Society, 14(5), 638-659. doi: http://dx.doi.org/10.1080/13691 $18 \times .2010 .514355$

Eshet-Alkalai, Y. (2012). Thinking in the digital era: A revised model for digital literacy. Issues in Informing Science and Information Technology, 9, 268-276.

Espinar, E. y González, M. J. (2009). Jóvenes en las redes sociales virtuales. Un análisis exploratorio de las diferencias de género. Feminismo/s, 14, 87-106. doi: http://dx.doi.org/10.14198/fem.2009.14.06

Gimeno, M. (Dir.) (2014). eEspaña 2014. Informe sobre el desarrollo de la sociedad de la información en España. Fundación Orange: Madrid.

Greenhow, C., y Robelia, E. (2009). Old Communication, New Illiteracies: Social Network Sites as Social Learning Resources. Journal of Computer-Mediated Communication, 14, 4, 1.130-1.161. doi: http:// dx.doi.org/10.1111/j.1083-6101.2009.01484.x

Hargittai, E. (2010). Digital Na (t) ives? Variation in Internet Skills and Uses among Members of the "Net Generation" Sociological Inquiry, 80(1), 92-113. doi: http://dx.doi.org/10.1111/j.1475-682X.2009.00317.x Hargittai, E. y Walejko, G. (2008). The participation divide: Content creation and sharing in the digital age. Information,Communication\&Society, 17(2),239-256.doi:http://dx.doi.org/10.1080/13691180801946150

Helsper, E. (2008). Digital inclusion: an analysis of social disadvantage and the information society. London: Department for Communities and Local Government.

Helsper, E. y Eynon, R. (2013). Distinct skill pathways to digital engagement. European Journal of Communication, 28(6).doi: http://dx.doi.org/10.1177/0267323113499113

Hoffmann, Ch. P., Lutz, Ch. y Meckel M. (2015). Content creation on the Internet: a social cognitive perspective on the participation divide. Information, Communication \& Society, 18(6), 696-716. doi: http:// dx.doi.org/10.1080/1369118X.2014.991343

Jenkins, H., Clinton, K., Purushotma, R., Robison, A. J., y Weigel, M. (2006). Confronting the Challenges of Participatory Culture: Media Education for the 21 Century. MacArthur Foundation.

Kimmons, R. y Veletsianos, G. (2014). The fragmented educator 2.0: Social networking sites. Acceptable identity fragments and the identity constellation. Computers \& Education, 72, 292-301. doi: http://dx. doi. 
org/10.1016/j.compedu.2013.12.001

Kimmons, R. (2014). Social networking sites, literacy, and the authentic identity problem. Tech Trends, 58(2), 93-98. doi: http://dx.doi.org/10.1007/s11528-014-0740-y

Lankshear, C., y Knobel, M. (2008). Digital literacies: Concepts, policies and practices. New York: Peter Lang.

Lara, T. (2011). Alfabetizar en la cultura digital. [Mensaje en Blog] Blog Tiscar.com. Extraído el 20 de marzo de 2014 de http://tiscar.com/2011/07/17/alfabetizar-en-la-cultura-digital/

Litt, E. (2013). Understanding social network site users' privacy tool use. Computers in Human Behavior, 29(4), 1649-1656. doi: http://dx.doi.org/10.1016/j.chb.2013.01.049

Livingstone, S. (2008). Engaging with media matter of literacy? Communication, Culture \& Critique, 7(1), 51-62. doi: http://dx.doi.org/10.1111/j.1753-9137.2007.00006.x

Martin, A., y Grudziecki, J. (2006). DigEuLit: Concepts and Tools for Digital Literacy Development. University of Glasgow, Scotland, 249-267. doi: http://dx.doi.org/10.11120/ital.2006.05040249

Menéndez, M. I. (2012). Cultura digital genérica. Usos y consumos de las mujeres. Telos, 91, 45-53

Mills, K. A. (2010). A review of the "digital turn" in the New Literacy Studies. Review of Educational Research, 80(2), 246-271. doi: http://dx.doi.org/10.3102/0034654310364401

Schradie, J. (2013). The Digital Production Gap in Great Britain. Information, Communication \& Society, 16(6), 989-998. doi: http://dx.doi.org/10.1080/1369118X.2013.799305

Van Deursen, A. J. A. M., y Van Dijk, J. A. G. M. (2016). Modeling Traditional Literacy, Internet Skills and Internet Usage: An Empirical Study. Interacting with Computers, 28(1), 13-26. doi: http://dx.doi. org/10.1093/iwc/iwu027

Van Deursen, A. J. A. M., Helsper, E., y Eynon R. (2014). Measuring Digital skills. From Digital Skills to Tangible Outcomes project report. London: University of Twente.

Van Deursen A. J. A. M., y Van Dijk J. A. G. M. (2010). Measuring internet skills. International Journal of Human-Computer Interaction, 26(10), 891-916. doi: http://dx.doi.org/10.1080/10447318.2010.496338

Van Dijk, J. (2006). Digital divide: Research, achievements and shortcomings. Poetics, 34, 221-235. doi: http://dx.doi.org/10.1016/j.poetic.2006.05.004

Van Dijk, J. (2005). The Deepening Divide Inequality in the Information Society. London: Sage Publications. 